\title{
Laryngitis, CTCAE
}

National Cancer Institute

\section{Source}

National Cancer Institute. Laryngitis, CT CAE. NCI Thesaurus. Code C143641.

A disorder characterized by an inflammatory process involving the larynx. 